\title{
Contribution of thermal cues to material discrimination and localization
}

\author{
HSIN-NI HO and LYNETTE A. JONES \\ Massachusetts Institute of Technology, Cambridge, Massachusetts
}

\begin{abstract}
The objective of these two experiments was to determine the role of thermal cues in material discrimination and localization, using materials that spanned a range of thermal properties. In the first experiment, the subjects were required to select the cooler of two materials presented to the index fingers. In the second, the finger that was in contact with a material that was different from that presented to the other two fingers on the same hand had to be identified. The results indicated that the subjects were able to discriminate between materials, using thermal cues, when the differences in their thermal properties were large. The changes in skin temperature when the fingers were touching the materials were, however, smaller than those predicted by the theoretical model. The ability to localize the thermal changes when three fingers on the same hand were stimulated was poor and depended on both the thermal properties of the target and the distractor materials.
\end{abstract}

The ability to identify and discriminate between objects by touch is based on the perception of a number of properties, including shape, surface texture, compliance, and thermal characteristics. These cues become particularly important when objects must be identified in the absence of vision. The human hand is capable of resolving remarkably fine variations in texture, as is shown by its capacity to detect periodically ordered elements that are only $0.06 \mu \mathrm{m}$ high when there is a relative motion between the texture and the finger pad (LaMotte \& Srinivasan, 1991). The ability to discriminate between the compliance of objects depends on whether the objects have deformable or rigid surfaces. With deformable surfaces, cutaneous cues from skin deformation are sufficient to discriminate compliance, whereas for rigid objects both cutaneous and proprioceptive cues are necessary for discrimination (Srinivasan \& LaMotte, 1995). The thermal cues that are used to assist in identifying an object arise from changes in skin temperature that occur when the object is held in the hand. Warm and cold thermoreceptors in the skin discharge in response to these local thermal transients. The resting temperature of the skin is generally higher than the temperature of the object in contact with the skin, and so it is the cold thermoreceptors that signal the decrease in skin temperature upon contact.

The ability to perceive thermal changes depends on many factors, including the amplitude and rate of temperature change, the baseline temperature of the skin, and the site

This research was supported in part through the Advanced Decision Architectures Collaborative Technology Alliance sponsored by the U.S. Army Research Laboratory under Cooperative Agreement DAAD19-012-0009 and by Grant NS-40836 from the National Institutes of Health. Correspondence concerning this article should be addressed to L. A. Jones, Department of Mechanical Engineering, Massachusetts Institute of Technology, Room 3-137, 77 Massachusetts Ave., Cambridge, MA 02139 (e-mail: ljones@mit.edu). stimulated (for reviews, see Darian-Smith, 1984; Stevens, 1991). The threshold for discriminating the difference in the amplitudes of two temperature pulses delivered to the thenar eminence of the hand is $0.02^{\circ}-0.07^{\circ} \mathrm{C}$ for cooling pulses and $0.03^{\circ}-0.09^{\circ} \mathrm{C}$ for warming pulses (Johnson, Darian-Smith, \& LaMotte, 1973; Johnson, Darian-Smith, LaMotte, Johnson, \& Oldfield, 1979). This is considerably lower than the threshold for discriminating a change in skin temperature. When the skin temperature of the thenar eminence is maintained at $33^{\circ} \mathrm{C}$, the differential threshold is $0.20^{\circ} \mathrm{C}$ for warming and $0.11^{\circ} \mathrm{C}$ for cooling (Stevens \& Choo, 1998). If skin temperature changes very slowly-for example, at a rate of less than $0.5^{\circ} \mathrm{C} / \mathrm{min}$ - an observer can be unaware of a change of up to $4^{\circ}-5^{\circ} \mathrm{C}$, provided that the temperature remains within the neutral thermal zone of $30^{\circ}-36^{\circ} \mathrm{C}$ (Kenshalo, 1976).

The resting temperature of the skin on the hand ranges from $25^{\circ}$ to $36^{\circ} \mathrm{C}$ (Verrillo, Bolanowski, Checkosky, \& McGlone, 1998) and typically is higher than the ambient temperature of materials encountered in the environment. The thermal cues used to identify a material by touch are influenced by the interface temperature and the heat flux conducted out of the skin upon contact. These are, in turn, a function of thermal properties, such as conductivity, heat capacity, and the initial temperatures of the skin and material. The thermal interaction between the skin and a material in contact with the skin is a transient process and is dominated by heat conduction. As long as the contact time is short enough for a semi-infinite body model to be valid (Lienhard \& Lienhard, 2003), this process can be modeled as two semi-infinite bodies in contact. On the basis of this model, the temperature of the skin surface upon contact can be calculated from Equation 1:

$$
T_{\mathrm{s}}=\frac{T_{\text {material, } \mathrm{i}}(k \rho c)_{\text {material }}^{1 / 2}+T_{\text {skin,i }}(k \rho c)_{\text {skin }}^{1 / 2}}{(k \rho c)_{\text {material }}^{1 / 2}+(k \rho c)_{\text {skin }}^{1 / 2}},
$$


where $T_{\mathrm{s}}$ is the temperature of the skin surface upon contact, $T_{\text {material, } \mathrm{i}}$ is the initial temperature of the material, $T_{\text {skin,i }}$ is the initial temperature of the skin, $k$ is the thermal conductivity, $\rho$ is density, $c$ is specific heat, and $(k \rho c)^{1 / 2}$ is the contact coefficient. The interface temperature, $T_{\mathrm{s}}$, depends on the thermal properties and initial temperatures of the skin and material. The thermal property that influences this process is the contact coefficient, $(k \rho c)^{1 / 2}$, and it acts as a weighting factor that determines whether $T_{\mathrm{s}}$ will more closely approach $T_{\text {skin,i }}$ or $T_{\text {material, } \mathrm{i}}$ (Incropera $\&$ DeWitt, 1996). The heat flux conducted out of the skin upon contact is time dependent in this process and can be calculated from Equation 2:

$$
q_{\text {skin }}^{\prime \prime}=\frac{-k_{\text {skin }}\left(T_{\mathrm{s}}-T_{\text {skin,i }}\right)}{\left(\pi \alpha_{\text {skin }} t\right)^{1 / 2}},
$$

where $q_{\text {skin }}^{\prime \prime}$ is the heat flux conducted out of the skin upon contact, $k_{\text {skin }}$ is the thermal conductivity of the skin, $\alpha_{\text {skin }}$ is the thermal diffusivity of the skin, and $t$ is time. Because the heat flux conducted out of skin depends on the interface temperature, $T_{\mathrm{s}}$, the decrease in skin temperature upon contact, $\Delta T$, [ $T_{\text {skin,i }}-T_{\mathrm{s}}$, can be used as an index of how cold the skin would feel when making contact with the material. When a person makes contact with a material, the contact coefficients of the skin and the material determine the interface temperature and the heat flux conducted out of the skin. This change in skin temperature is encoded by thermoreceptors in the dermis and is transmitted to the central nervous system to assist in object identification.

The Fourier number of the material can be used to determine whether it can meet the requirement of the semiinfinite body model. The definition of the Fourier number, $F_{0}$, is

$$
F_{\mathrm{o}}=\frac{\alpha t}{L_{\mathrm{c}}^{2}}
$$

where $\alpha$ is thermal diffusivity of the material, $L_{\mathrm{c}}$ is characteristic length, which is defined as the material volume divided by the contact area, and $t$ is time. Generally speaking, a semi-infinite body model is valid under the condition that the Fourier number of the material in the process is less than 0.05 (Mills, 1999). For materials whose Fourier numbers are larger than 0.05 , the semi-infinite body model can still serve as a reasonable description of the transient thermal process.

Several studies have suggested that subjects can identify objects varying in thermal properties, using only thermal cues. Caldwell and Gosney (1993) recorded the thermal changes on a teleoperated robotic hand as it made contact with a variety of objects, including an ice cube, a heated soldering iron, an aluminum block, and a piece of insulation foam, and then presented these thermal transients to subjects. A signal from a temperature sensor (thermocouple) on the robotic hand maintained at $40^{\circ} \mathrm{C}$ indicated the type and magnitude of the thermal transient associated with contact with the various materials. These thermal sig- nals were then presented to the subjects, using a Peltier heat pump that was mounted within a finger sleeve of a glove that rested on the dorsal surface of the index finger. The subjects achieved an $80 \%$ success rate in identifying the various materials on the basis of these thermal cues only.

In a further study of thermal cues and object identification, Ino et al. (1993) measured the decrease in finger temperature as subjects made contact with a number of materials that they were required to identify. Of the five materials presented to the fingertip, the subjects could reliably identify aluminum and wood, with success rates higher than $80 \%$, but confused glass, rubber, and polyacrylate. The decrease in finger temperature as the hand made contact with the materials was then used to simulate the thermal transients associated with contact. The thermal simulations were presented using a display that comprised a Peltier element, a thermocouple, and a proportional integral and derivative controller. The subjects were required to identify which of the five materials was being presented, using only the thermal cues presented on the fingertip. Ino et al. found that the recognition rates for the various samples presented with the thermal display were equivalent to those measured using real materials and that there was no significant difference in the information transmission rates associated with the real object and the Peltier-based thermal display. In both of these studies (Caldwell \& Gosney, 1993; Ino et al., 1993), the subjects were required to identify the material on the basis of the pattern of thermal stimulation presented to the hand, and the subjects knew in advance the types of material being presented. These results suggest that subjects can use thermal cues effectively to identify objects and that different materials have thermal transients associated with skin contact that are perceptually distinct.

The goals of the present set of experiments were to determine the nature of the thermal cues used to discriminate between materials and to evaluate the relation between the actual and the theoretical changes in skin temperature as materials with different thermal properties made contact with the fingers. In the first experiment, the subjects were required to discriminate between two materials presented to the left and right index fingers, and in the second experiment, the task was to identify which of three fingers on the same hand was in contact with a material that was different from the other two. In the latter experiment, the ability to localize thermal changes was evaluated.

\section{EXPERIMENT 1 Material Discrimination}

\section{Method}

Subjects. Ten normal healthy adults ( 5 women and 5 men), between 20 and 35 years of age, participated in this experiment. They included undergraduate and graduate students and research staff in the Department of Mechanical Engineering at MIT. They had no known abnormalities of the tactile or thermal sensory systems and no history of peripheral vascular disease. All of them reported that they were right-handed. This research was approved by the local ethics committee. 
Apparatus. Six materials that covered a broad range of thermal properties were selected: copper, bronze, stainless steel, glass reinforced epoxy (G10), plastic (ABS), and foam. Their thermal properties are listed in Table 1. On the basis of the semi-infinite body model, the corresponding skin temperature upon contact with the materials is shown in Table 1 and Figure 1A, and the corresponding heat flux conducted out of the skin with contact is shown in Figure 1B.

The materials were stored at room temperature, which was maintained at $24^{\circ} \mathrm{C}$. Each sample was $12.4 \mathrm{~mm}$ in diameter and $100 \mathrm{~mm}$ long and had a flat upper surface that was $12 \times 60 \mathrm{~mm}$. They were turned from $12.7-\mathrm{mm}(0.5$-in.) rod stock, milled, and then sanded to provide a flat, smooth contact surface. Their surface roughness was measured with a Mitutoyo Surface Roughness Tester (Model SV-3000S4). The surface roughness was measured to ensure that textural differences among the material samples were minimized. These values and the Young's modulus (the tensile elastic modulus) of the materials are listed in Table 2.

Two pieces of delrin were used to make a material presentation fixture, as is shown in Figure 2. The combined size of these pieces when screwed together was $103 \times 63 \times 46 \mathrm{~mm}^{3}$. Two $22 \times 20 \mathrm{~mm}^{2}$ rectangular holes were machined into the upper piece to allow insertion of the fingers. In the lower piece of delrin, two $12.5-\mathrm{mm}$ diameter compartments were machined $43 \mathrm{~mm}$ apart directly under the holes. The material samples slid into these slots and were flush with the surrounding surface. An extended roof was added on top of the fixture to prevent the subjects from seeing the material samples.

A thermistor ( $457 \mu \mathrm{m}$ in diameter and $3.18 \mathrm{~mm}$ in length; Model 56A1002-C8, Alpha Technics) measured the skin temperature of the index finger. The thermistors were connected to a data acquisition unit (Model 34970A, Agilent Technologies), which was controlled using a Visual Basic program. Temperature data were sampled at $20 \mathrm{~Hz}$. To ensure that the skin temperature of the hand was maintained constant, a fixture was made using a recirculating chiller (Model 1167P, VWR International). The chiller was connected to a spiral folded tube (ID $4.8 \mathrm{~mm}$, OD $7.9 \mathrm{~mm}$ ) that was placed in a delrin fixture. A 2-mm-thick copper plate was placed on the tube to improve the temperature uniformity of the surface. A 3.2-mm-thick rubber pad was then added on top of the copper plate to increase comfort for the subjects when they placed their hands on the fixture to maintain skin temperature

Procedure. The subjects washed their hands with soap prior to participating in the experiment. A thermistor was glued to the finger pads of each index finger, using biocompatible cyanoacrylate (Liquid Bandage, Johnson \& Johnson). The thermistor was $457 \mu \mathrm{m}$ in diameter and $3.18 \mathrm{~mm}$ in length and was chosen on the basis of its small dimensions and thermal mass. To ensure that skin temperature measurements were not influenced by contact force directly, baking soda was used to mark the contact area of the skin with the material, and the thermistor was glued onto the edge of the contact area. The subjects' initial skin temperatures ranged from $30^{\circ}$ to $35.5^{\circ} \mathrm{C}$, and the average value was $33^{\circ} \mathrm{C}$. The ambient temperature in the room was maintained at $24^{\circ} \mathrm{C}$, as measured with a k-type thermocouple (Omega) in free air.

Each of the six materials was paired with all the other materials, including itself, which gave a total of 21 different combinations. These 21 combinations were repeated four times, for a total of 84 trials. Within each block of 21 trials, the order of presentation of the materials was randomized. There was at least a 1-min break between each block of trials, during which the subjects placed their hands on the recirculating chiller.

Prior to each trial, the subjects were instructed to place both of their hands on the recirculating chiller to maintain their skin temperature at $33^{\circ} \mathrm{C}$. For the first part of the experiment, two material samples were inserted into the two separate compartments, as is shown in Figure 2. The subjects inserted their left and right index fingers into the compartments after hearing a sound cue. The data acquisition system recorded temperature from the thermistors attached to the subjects' finger pads after the sound cue. At the end of each trial, the subjects withdrew their hands from the fixture and placed them on the recirculating chiller pad. A maximum of $10 \mathrm{sec}$ was allowed for each trial.

A two-alternative forced choice method was used in which the subjects were instructed to choose the colder of the two materials presented by reporting which hand made contact with the colder material. The subjects were not told which materials were used, and no feedback was given regarding the correctness of their judgments. They were encouraged to lift and replace their fingers on the material samples during each trial but were discouraged from lateral scanning of the sample surface.

The subjects lifted and replaced their fingers on the material repeatedly during each trial, which meant that the temperature responses fluctuated and, so, it was difficult to record meaningful temperature data. The measurement of the decrease in skin temperature on contact was therefore undertaken separately. The procedure in this second part of the experiment was similar to that in the first, except that the subjects were instructed to leave their index fingers on the sample surface for $5 \mathrm{sec}$ after inserting their left and right index fingers into the compartments. The goal of this part of the experiment was to measure the change in skin temperature upon contact.

\section{Results}

In the first experiment, the responses for the trials involving different materials were analyzed in terms of the number of correct responses - that is, correctly identifying the "colder" of the two materials, as defined in terms of the predicted thermal responses at the skin-surfacematerial interface. The chance level in this experiment was $50 \%$, and a threshold level of $72 \%$ correct was chosen as indicating that subjects could reliably discriminate between a pair of materials. An initial analysis of the results

Table 1

Thermal Properties of the Materials

\begin{tabular}{|c|c|c|c|c|c|c|}
\hline Material & $\begin{array}{c}\text { Conductivity } k \\
(\mathrm{~W} / \mathrm{mK})\end{array}$ & $\begin{array}{c}\text { Density } \rho \\
\left(\mathrm{kg} / \mathrm{m}^{3}\right)\end{array}$ & $\begin{array}{c}\text { Specific Heat } c \\
(\mathrm{~J} / \mathrm{kgK})\end{array}$ & $\begin{array}{c}\text { Contact } \\
\text { Coefficient }(k \rho c)^{1 / 2} \\
\left(\mathrm{~J} / \mathrm{m}^{2} \mathrm{~s}^{1 / 2} \mathrm{~K}\right)\end{array}$ & $\begin{array}{c}\text { Skin Temperature } \\
\text { Upon Contact } \mathrm{T}_{\mathrm{s}} \\
\left({ }^{\circ} \mathrm{C}\right)^{*}\end{array}$ & $\begin{array}{c}\text { Fourier } \\
\text { Number } \\
F_{0}^{\dagger} \\
\end{array}$ \\
\hline Copper & 398.000 & 8,954 & 384 & 36,992 & 24.3 & 3.580 \\
\hline Bronze & 54.000 & 8,780 & 355 & 12,973 & 24.7 & 0.540 \\
\hline Stainless steel & 13.500 & 8,000 & 460 & 7,048 & 25.3 & 0.110 \\
\hline G10 & 0.290 & 1,800 & 1,600 & 910 & 29.0 & 0.003 \\
\hline ABS & 0.180 & 1,010 & 1,386 & 501 & 30.3 & 0.004 \\
\hline Foam & 0.029 & 24 & 1,210 & 29 & 32.8 & 0.030 \\
\hline
\end{tabular}

Note - G10 is a glass-reinforced epoxy, and ABS is a plastic. ${ }^{*}$ Based on a semi-infinite body model with initial skin temperature at $33^{\circ} \mathrm{C}$ and initial material temperature at $24^{\circ} \mathrm{C}$. ${ }^{\dagger}$ Based on a 5 -sec contact time and sample dimensions. 
(A)

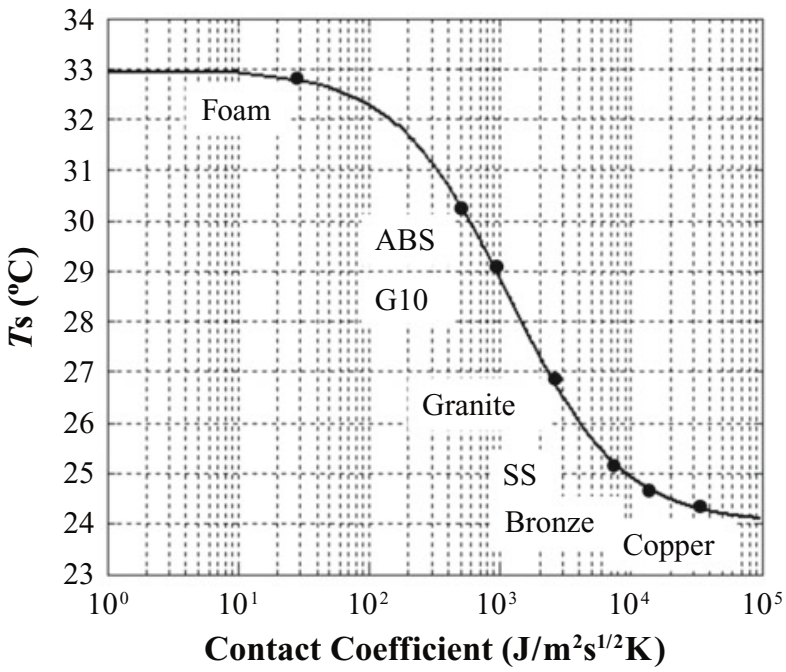

(B)

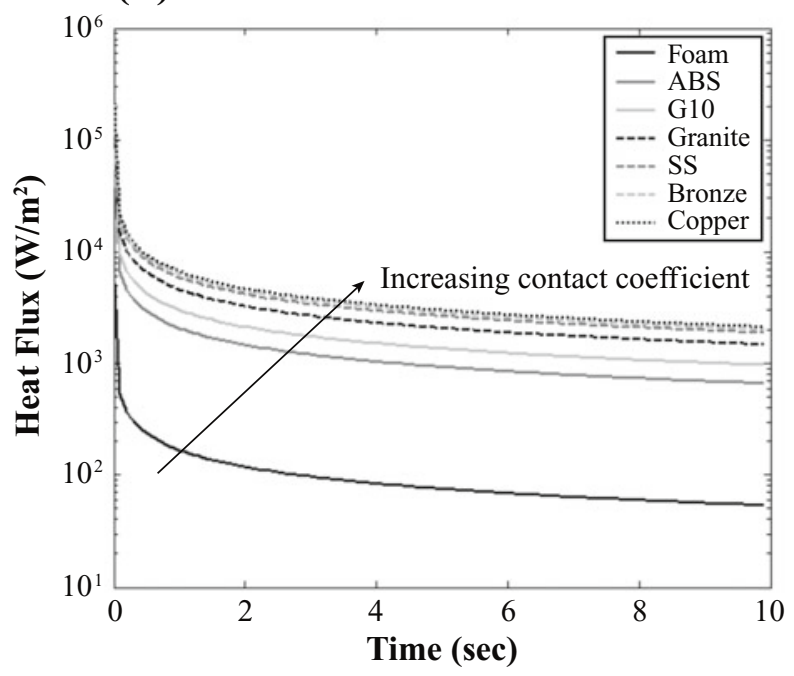

Figure 1. (A) Skin temperatures and (B) corresponding heat fluxes conducted out of the skin upon contact with the identified material, based on the semi-infinite body model. ABS, a plastic; G10, a glass-reinforced epoxy; SS, stainless steel.

from trials in which the same material was presented to both hands indicated that there was a bias toward reporting that the material on the right index finger was colder $(t=2.08, p=.04)$. This did not reflect any temperature difference between the hands, since both were maintained at $33^{\circ} \mathrm{C}$.

The percentage of correct discriminations for the various combinations of materials is shown in Table 3 . For all combinations except copper-bronze and bronze-stainlesssteel, the percentages were above $72 \%$. This means that the subjects were able to discriminate reliably between two materials when the ratio of the contact coefficients of the materials exceeded three. For combinations involving foam, the percentage of correct discriminations ranged from $85 \%$ to $100 \%$. This high performance presumably reflects the distinct thermal and textural properties of foam - in particular, its much greater surface roughness when compared with the other materials, as is shown in Table 2. ANCOVAs were conducted to determine whether the surface roughness and elasticity of the materials (the latter defined in terms of Young's modulus, which is the ratio of the stress to strain) influenced the subjects' performance. The covariates were the ratio of the surface roughness of the two materials being compared or the ratio of their Young's moduli. The analyses indicated that there was no significant effect of the ratio of the materials' surface roughness $(p=.53)$ or Young's modulus $(p=.20)$ on the percentage of correct responses.

In the second part of the experiment, the change in temperature upon contact was recorded for each of the materials presented. These data are shown in Figure 3, where it can be seen that the decrease in skin temperature was small, ranging from a mean of $0.25^{\circ}$ for foam to $1.44^{\circ} \mathrm{C}$ for bronze. A one-way ANOVA indicated that there was no significant difference in the decrease in skin temperature across the six materials presented $[F(5,48)=1.927, p=.11]$. With the exception of foam, the decreases in skin temperature as the finger made contact with the six materials were considerably smaller than the theoretical values listed in Table 1, which were calculated using an initial skin temperature of $33^{\circ} \mathrm{C}$ and an initial material temperature of $24^{\circ} \mathrm{C}$.

\section{Discussion}

In this material discrimination task, the subjects perceived thermal cues by lifting and replacing their fingers on the surfaces, and the local thermal transients associated with contact were presumably used to discriminate between the materials. The results from this experiment indicate that when textural cues are minimized, thermal cues can be used to discriminate between materials when the differences in the contact coefficients are relatively large. The only two combinations that could not be reliably discriminated were copper and bronze, and bronze and stainless steel. The thermal cues used to discriminate between these materials, such as the heat fluxes conducted out of the skin, were so similar that they did not facilitate discrimination. These results are consistent with the

Table 2

Surface Roughness and Young's Modulus of the Material Samples

\begin{tabular}{lcc}
\hline Material* & $\begin{array}{c}\text { Mean Rq } \\
(\mu \mathrm{m})\end{array}$ & $\begin{array}{c}\text { Young's Modulus } \\
(\mathrm{GPa})\end{array}$ \\
\hline Copper & 0.40 & 110.0 \\
Bronze & 0.32 & 93.0 \\
SS & 0.43 & 193.0 \\
ABS & 0.20 & 2.3 \\
G10 & 1.50 & 17.0 \\
Granite & 1.47 & 53.0 \\
Foam & 15.22 & $1.50 \mathrm{E}-03$ \\
\hline
\end{tabular}

*Copper, bronze, stainless steel (SS), a glass-reinforced epoxy (G10), a plastic (ABS), and foam were used in the first experiment. Copper, stainless steel, ABS, granite, and foam were used in the second experiment. $\dagger \mathrm{Rq}$, root mean square amplitude of the irregularities on the surface. 

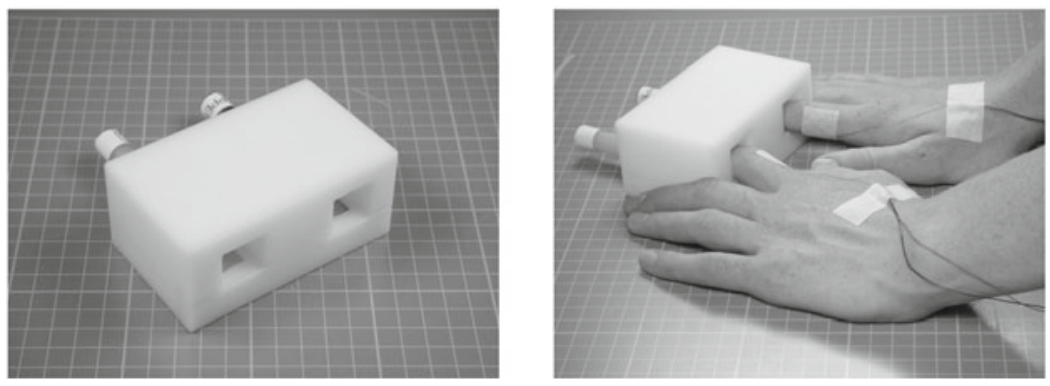

Figure 2. Material discrimination fixture. The thermistors are glued to the index fingers.

semi-infinite body model illustrated in Figures 1A and $1 \mathrm{~B}$, which show the similarity in the predicted heat fluxes for copper and bronze and for bronze and stainless steel.

Although the thermal cues associated with contact were presumably used to discriminate between the materials, differences in surface texture and elasticity could have influenced the subjects' performance. However, the analyses indicated that the percentage of correct responses was not significantly affected by the ratio of the surface roughness or the Young's moduli of the materials being compared. The ease with which foam was discriminated when presented along with any of the other materials is probably due to its distinctive thermal characteristics, surface roughness, and elasticity. Of all the materials presented, foam changed the skin temperature the least. The average decrease in skin temperature when contact was made with foam was $0.25^{\circ} \mathrm{C}$, which is above the threshold of $0.14^{\circ} \mathrm{C}$ for discriminating decreases in skin temperature on the fingertip (Stevens \& Choo, 1998). Although the surface roughness and compliance of foam may have provided additional cues to assist in material discrimination, the percentages of correct discriminations for the combinations including foam decreased progressively from copper to ABS. This suggests that even with the extra tactile cues, the ability to discriminate foam from the other materials was influenced by thermal properties.

The elastic moduli (i.e., Young's modulus) of the materials varied considerably, with foam being the most elastic. The typical contact force during this type of thermal discrimination experiment would have been between 1.5 and $2 \mathrm{~N}$ (Jones \& Berris, 2003), and the average contact

Table 3

Percentages of Correct Discriminations for Different Pairs of Materials

\begin{tabular}{lccccrc}
\hline & Copper & Bronze & SS & G10 & ABS & Foam \\
\hline Copper & 70 & 75 & 95 & 98 & 100 \\
Bronze & & & 68 & 98 & 98 & 100 \\
SS & & & & 98 & 100 & 95 \\
G10 & & & & & 78 & 90 \\
ABS & & & & & & 85 \\
Foam & & & & & & \\
\hline
\end{tabular}

Note-SS, stainless steel. G10 is a glass-reinforced epoxy, and ABS is a plastic. area was $135 \mathrm{~mm}^{2}$. On the basis of these force and contact area estimates and the Young's modulus for each material, the strain of the materials (i.e., the change in length of the material normalized by the initial length) was calculated. The estimated strain for foam is 0.01 , and for the other materials it ranged between $10^{-6}$ and $10^{-7}$. From these values, it would seem reasonable to assume that, with the exception of foam, no deformation occurred during contact with these materials.

The present findings are consistent with those of Dyck, Curtis, Bushek, and Offord (1974), who designed a set of thermal stimulators known as the Minnesota thermal disks. These disks are made from copper, stainless steel, glass, and polyvinyl chloride (PVC) and were developed as a clinical tool to measure thermal sensation. Dyck et al. found that the pairs of disks that normal healthy subjects reliably distinguished on the palm of the hand as "cold" and "warm" were copper and PVC, and copper and glass. The contact coefficients of copper, PVC, and glass are $36,992,406$, and $1,510 \mathrm{~J} / \mathrm{m}^{2} \mathrm{~s}^{1 / 2} \mathrm{~K}$, respectively. Both of the contact coefficient ratios of the pairs that Dyck et al. reported were distinguished are greater than three, in agreement with the present findings.

The decreases in skin temperature upon contact with the materials (shown in Figure 3) were considerably smaller than the theoretical values predicted from the semiinfinite body model (Table 1). The difference between these two sets of values results from the fact that skin is not a "semi-infinite" inanimate object, as is assumed in the semi-infinite body model, but has an internal source of heat generation. Moreover, the thermal properties of skin make it a good thermal insulator $\left(\alpha=10^{-7} \mathrm{~m}^{2} / \mathrm{sec}\right)$, which means that changes in temperature can be localized to a small region of skin (Eberhart, 1985). Because of the localized nature of the change in skin temperature, the thermistor attached to the edge of the contact area may not have detected the full extent of the temperature change. The absence of any significant difference in the decrease in skin temperature after contact with the various materials also reflects the variability between subjects in the measured thermal changes. Factors such as the volume and width of the finger pad, the contact area, and the contact force can all affect the thermal response of the finger (Jones \& Berris, 2003). 


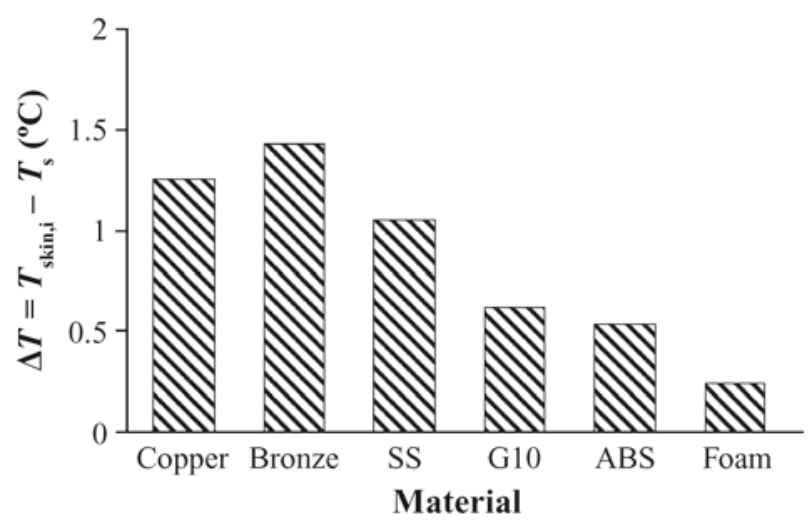

Figure 3. Group mean decrease in skin temperature after $5 \mathrm{sec}$ of contact with the six materials. SS, stainless steel; G10, glass reinforced epoxy; ABS, a plastic.

When the finger makes contact with an object, the contact area begins as a single point and expands exponentially in size with surface compression. The contact distribution is symmetric in the medial-lateral direction, but not in the proximal-distal direction (Pawluk \& Howe, 1999). The force imposed by a finger on an object can affect thermal responses in two ways. First, compression of the cutaneous tissue of the index finger may enhance thermal sensing by increasing the area of contact with the object. Second, compression can affect finger temperature by collapsing blood vessels in the region, which prevents continuous tissue-heat exchange. Of the blood flow to the fingertip, $90 \%$ is considered to be involved in thermal regulation, with the remaining $10 \%$ required for nutrition (Tubiana, 1981). Although the digital arteries, which are protected by the underlying bone, are unaffected by the pressure exerted by the finger pad, the larger, more compliant digital veins that run lateral to the bone have a lower internal blood pressure and are more susceptible to collapse with pressure. This results in an accumulation of blood in the capillaries under the nail bed (Wolfram-Gabel \& Sick, 1995) and an increase in skin temperature. This may also contribute to the smaller than predicted decreases in temperature upon contact with the various materials.

The semi-infinite body model was used to predict the interaction between the skin and the materials, and the Fourier numbers of the materials in contact with the skin for $5 \mathrm{sec}$ were calculated (see Table 1). The Fourier numbers for G10, ABS, and foam confirmed that the semi-infinite body model was valid for them, but copper, bronze, and stainless steel all had Fourier numbers well above 0.05 . Thus the semi-infinite body model can give only an approximate description of the skin-material interaction for these materials.

Although the measured decreases in temperature upon contact with the materials in this experiment were small, ranging from $0.25^{\circ}$ to $1.44^{\circ} \mathrm{C}$, they were presumably perceived by the subjects. Psychophysical studies of thermal thresholds indicate that at a skin temperature of $33^{\circ} \mathrm{C}$, the threshold for detecting a decrease in temperature is $0.14^{\circ} \mathrm{C}$ on the tip of the index finger and $0.11^{\circ} \mathrm{C}$ on the thenar eminence (Stevens \& Choo, 1998). These threshold values are smaller than the thermal changes measured as the index finger made contact with the materials. The time course and amplitude of the thermal responses are, however, markedly different from those reported by Ino et al. (1993), who, for a single subject, showed an immediate decline in skin temperature upon contact with all materials. The decreases in skin temperature that they reported ranged from $0.1^{\circ} \mathrm{C}$ for wood to $7^{\circ} \mathrm{C}$ for aluminum and occurred within $1 \mathrm{sec}$ and then stabilized within $500 \mathrm{msec}$, which is an extremely rapid and dramatic response for the peripheral thermal system. Caldwell and Gosney (1993) reported that it took 3-5 sec to obtain a successful thermal reading from the hand as it made contact with a range of materials, and in the present study the change in skin temperature did not stabilize for $5 \mathrm{sec}$. The latter data are consistent with the long reaction and decision times reported for thermal stimuli (Lederman \& Klatzky, 1997; Stevens, 1991).

\section{EXPERIMENT 2 Localization of Thermal Cues From Different Materials}

A second experiment was conducted to examine how accurately subjects can localize a thermal stimulus in the presence of other thermal stimuli on the same hand and to determine whether this changes as a function of the location of the target stimulus and the thermal properties of the stimuli. The thermal perceptual system has been shown to have poor spatial localization but good spatial summation for both warm (Hardy \& Oppel, 1937; Marks \& Stevens, 1973; Marks, Stevens, \& Tepper, 1976) and cold (Hardy \& Oppel, 1938; Stevens \& Marks, 1979) stimuli, which means that identifying where a specific thermal change occurs is difficult. The rules governing spatial summation for warmth and cold differ, in that the degree of spatial summation of warmth declines with increasing stimulus intensity, whereas the summation of cold tends to remain about the same with decreases in temperature ranging from $1.5^{\circ}$ to $12^{\circ} \mathrm{C}$ (Stevens \& Marks, 1979). The localization of a thermal change can be facilitated by the tactile input associated with contact and, for warm stimuli, improves with increasing intensity of stimulation (Simmel \& Shapiro, 1969). However, the interaction of thermal and tactile inputs can lead to mislocalization of thermal sensations when adjacent parts of the skin are differentially stimulated. Green (1977) described a thermal illusion involving the hand in which the thermal sensation experienced by the middle finger changed as a function of the sensations experienced at the two adjacent fingers. When the index and ring fingers were placed on cold (or warm) thermal stimulators and the middle finger was placed on a thermally neutral stimulator, cold (or warmth) was felt on all three fingers. The perceived magnitude of these thermal sensations was the same as that experienced in the control condition, in which the temperature of the thermal stimulator under the middle finger was varied and the outer two 
stimulators remained thermally neutral. This referral of thermal sensations required equivalent tactile experiences on the three fingers, in that it did not occur when the middle finger was held above the stimulator (Green, 1977). These interactions between thermal and tactile inputs and spatial localization of thermal changes were the subject of the second experiment. Of particular interest was whether the subjects could localize a thermal event on the basis of relatively small changes in the skin temperature of the fingers as they contacted different materials and whether this depended on which finger was differentially stimulated.

In the present experiment, a range of materials with different thermal properties was used to induce thermal responses, and the subjects were required to indicate which of three materials felt different from the other two. The materials were presented to the index, middle, and ring fingers of the right hand, and the position of the target material varied from trial to trial. It was predicted that the subjects would have more difficulty in discriminating the target material in the presence of the other two stimuli (i.e., distractor material), as compared with the first experiment, in which only two stimuli were presented on each trial, and that the differences in the thermal properties of the materials would need to be significantly larger for correct performance.

\section{Method}

Subjects. Ten normal healthy adults ( 5 women and 5 men), between 20 and 29 years of age, participated in this experiment. Two of them participated in the first experiment. The time between the two experiments was over 6 months. The subjects had no known abnormalities of the tactile or thermal sensory systems and no history of peripheral vascular disease. They all reported that they were righthanded. This research was approved by the local ethics committee.

Apparatus. Five materials were selected for this experiment. They were copper, stainless steel, granite, plastic (ABS), and foam. In order to have a set of materials that spanned the full range of thermal properties (see Figure 1A), G10 was replaced by granite, and bronze was no longer used. The thermal properties are listed in Table 4. The material samples were stored at room temperature, which was maintained at $24^{\circ} \mathrm{C}$. Each sample was $19.05 \mathrm{~mm}$ wide, $145 \mathrm{~mm}$ long, and $38 \mathrm{~mm}$ thick. These sample dimensions were chosen to make the Fourier numbers of all the materials smaller than 0.05 , in order to meet the assumption of the semi-infinite body model. The surfaces of the samples were milled and then sanded, to provide a flat, smooth contact surface. The surface roughness of each material was measured to ensure that differences in surface texture among the material samples were minimized; these data are listed in Table 2.
Two pieces of delrin were used to make a material presentation fixture, as is shown in Figure 4. The combined size of these pieces when screwed together was $100 \times 100 \times 90 \mathrm{~mm}^{3}$. One $84 \times 45 \mathrm{~mm}$ rectangular pocket was machined into the upper piece to allow insertion of the index, middle, and ring fingers. Three $19.8 \times 95 \times$ $34 \mathrm{~mm}^{3}$ rectangular slots were machined into the lower piece. The material samples slid into these slots, and their surfaces were $4 \mathrm{~mm}$ above the surrounding surface. When inserting their fingers into the fixture, the subjects' three fingers were able to make contact with the three samples. An extended roof and an acrylic housing were added around the fixture, to prevent the subjects from seeing the material samples. To ensure that the skin temperature of the hand remained constant, the fixture with the recirculating chiller described in the previous experiment was also used in this experiment.

Procedure. The subjects washed their hands with soap prior to participating in the experiment. The subjects' initial skin temperatures ranged from $27^{\circ} \mathrm{C}$ to $36^{\circ} \mathrm{C}$, and the average value was $33.4^{\circ} \mathrm{C}$. The ambient temperature in the room was maintained at $24^{\circ} \mathrm{C}$, as measured with a k-type thermocouple (Omega) in free air.

Each of the five materials was paired with all other materials to give a total of 20 target-distractor combinations. There were three target positions - index, middle, and ring fingers - which gave 60 different combinations. These 60 combinations were then repeated three times, for a total of 180 trials. Within each block of 60 trials, the order of presentation of the materials was randomized. There was at least one 60 -sec break between each block of trials, during which the subjects placed their hands on the recirculating chiller. The experiment lasted about $90 \mathrm{~min}$.

Prior to each trial, the subjects were instructed to place their right hands on the recirculating chiller to maintain their skin temperature at $33^{\circ} \mathrm{C}$. Three material samples were inserted into the three rectangular slots, as is shown in Figure 4. The subjects inserted their index, middle, and ring fingers into the fixture after hearing a sound cue. A three-alternative forced choice (3AFC) method was used, in which the subjects were instructed to choose which finger felt different from the other two in terms of thermal changes on the finger pad. They were asked to report the number of the corresponding finger: 1 for the index finger, 2 for the middle finger, and 3 for the ring finger. The subjects were not told which materials were used for the samples, and no feedback was given regarding the correctness of their judgments. They were encouraged to lift and replace their fingers on the material samples but were discouraged from lateral scanning of the sample surface. The maximum time for each trial was $10 \mathrm{sec}$. After $10 \mathrm{sec}$, the experimenter asked the subjects to remove their hands from the fixture and place them back on the recirculating chiller.

\section{Results}

In this experiment, the responses for the trials were analyzed in terms of the number of correct responses - that is, correctly identifying the location of the target in the presence of two identical distractors. The chance level in this

Table 4

Thermal Properties of the Materials

\begin{tabular}{lcccccc}
\hline \multicolumn{1}{c}{ Material } & $\begin{array}{c}\text { Conductivity } k \\
(\mathrm{~W} / \mathrm{mK})\end{array}$ & $\begin{array}{c}\text { Density } \rho \\
\left(\mathrm{kg} / \mathrm{m}^{3}\right)\end{array}$ & $\begin{array}{c}\text { Specific Heat } c \\
(\mathrm{~J} / \mathrm{kgK})\end{array}$ & $\begin{array}{c}\text { Contact } \\
\text { Coefficient }(k \rho c)^{1 / 2} \\
\left(\mathrm{~J} / \mathrm{m}^{2} \mathrm{~s}^{1 / 2} \mathrm{~K}\right)\end{array}$ & $\begin{array}{c}\text { Skin Temperature } \\
\text { Upon Contact } T_{\mathrm{s}} \\
\left({ }^{\circ} \mathrm{C}\right)^{*}\end{array}$ & $\begin{array}{c}\text { Fourier } \\
\text { Number }\end{array}$ \\
\hline Copper & 398.000 & 8,954 & 384 & 36,992 & 24.3 & 0.0500 \\
Stainless steel & 13.500 & 8,000 & 460 & 7,048 & 25.3 & 0.0016 \\
Granite & 2.790 & 2,630 & 775 & 2,384 & 27.5 & $<0.0010$ \\
ABS & 0.180 & 1,010 & 1,386 & 501 & 30.3 & $<0.0010$ \\
Foam & 0.029 & 24 & 1,210 & 29 & 32.8 & $<0.0010$ \\
\hline
\end{tabular}

Note-ABS is a plastic. ${ }^{*}$ Based on a semi-infinite body model with initial skin temperature at $33^{\circ} \mathrm{C}$ and initial material temperature at $24^{\circ} \mathrm{C}$. ${ }^{\dagger}$ Based on 10 -sec contact time and the sample dimension. 


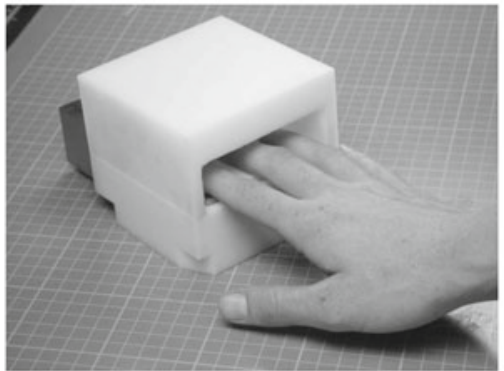

(A)

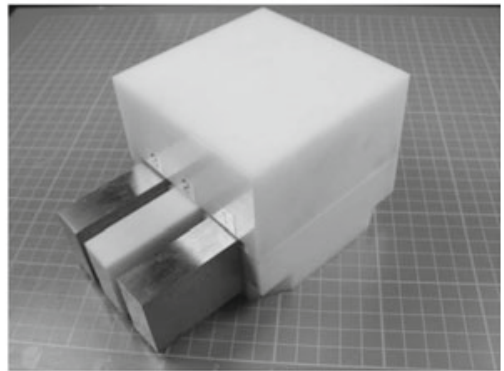

(B)

Figure 4. Front (A) and rear (B) views of the material presentation fixture used in Experiment 2.

experiment was $33 \%$. A test of proportion indicated that at a $54 \%$ level of correct performance, subjects can discriminate the target from the distractors reliably $(p<.0001)$. The percentages of correct responses were converted into the corresponding $d^{\prime}$ values for a 3AFC method (Gescheider, 1997). The percentage of trials in which the target location was correctly identified and the corresponding sensitivity, $d^{\prime}$, are shown in Table 5. A comparison of the results shown in Tables 3 and 5 indicates that the second experiment was considerably harder for the subjects than the first, with the overall correct response rate averaging $57 \%$, as compared with $90 \%$ in the first experiment. Performance was much poorer even for materials that were perfectly discriminated in the first experiment (e.g., copper and foam, and stainless steel and ABS).

In this experiment, the material presentation mode was divided into high/low and low/high, where high/low describes those trials in which the contact coefficient of the target material was higher than that of the distractor and low/high mode refers to those trials in which the contact coefficient of the target material was lower than that of the distractor. In the first condition, the target would feel colder than the two distractors, whereas in the latter condition, the distractors would feel colder than the target.

The results in Table 5 indicate that when the target had a higher contact coefficient than the distractor, the subjects were able to identify the target's location reliably when the ratio of the contact coefficients of the target and the distractor was higher than 14. For the low/high mode, three out of the four combinations that were reliably discriminated included foam as the target material, and the contact coefficient ratios for these combinations were all higher than 82. These results indicate that it is considerably easier to identify which finger is cooling the most than to select the finger that has the smallest change in skin temperature.

The group mean sensitivity, $d$, in identifying the location of the target as a function of whether the material was a target or a distractor is shown in Figure 5. As can be seen in the figure, the subjects performed better when presented with targets with extreme thermal properties (high or low contact coefficients) than when presented with those with median thermal properties. A repeated measures ANOVA of these values, with materials and pre-

Table 5

Percentage of Correct Responses, $p(c)$ and the Corresponding Average Sensitivity of the Subjects, $d$, for the Various Combinations of Materials, Regardless of Target Position

\begin{tabular}{|c|c|c|c|c|c|c|c|}
\hline \multirow[b]{2}{*}{ Material Combination } & \multirow{2}{*}{$\begin{array}{c}\text { Contact Coefficient } \\
\text { Ratio }\end{array}$} & \multicolumn{2}{|c|}{ High/Low } & \multicolumn{2}{|c|}{ Low/High } & \multicolumn{2}{|c|}{ Total } \\
\hline & & $p(c)$ & $d^{\prime}$ & $p(c)$ & $d^{\prime}$ & $p(\mathrm{c})$ & $d^{\prime}$ \\
\hline Stainless steel/granite & 2.96 & 30 & -0.12 & 43 & 0.33 & 37 & 0.13 \\
\hline Granite/ABS & 4.76 & 53 & 0.65 & 44 & 0.36 & 49 & 0.52 \\
\hline Copper/stainless steel & 5.25 & 38 & 0.16 & 39 & 0.20 & 38 & 0.16 \\
\hline Stainless steel/ABS & 14.07 & 72 & 1.31 & 59 & 0.85 & 66 & 1.09 \\
\hline Copper/granite & 15.52 & 56 & 0.75 & 44 & 0.36 & 50 & 0.56 \\
\hline $\mathrm{ABS} /$ foam & 17.28 & 68 & 1.16 & 46 & 0.43 & 57 & 0.78 \\
\hline Copper/ABS & 73.83 & 69 & 1.20 & 51 & 0.59 & 60 & 0.89 \\
\hline Granite/foam & 82.21 & 68 & 1.16 & 61 & 0.92 & 64 & 1.02 \\
\hline Stainless steel/foam & 243.03 & 79 & 1.61 & 62 & 0.95 & 71 & 1.28 \\
\hline Copper/foam & $1,275.59$ & 82 & 1.75 & 66 & 1.09 & 74 & 1.39 \\
\hline
\end{tabular}

Note-High/low refers to those trials in which the first material listed in column 1 was the target and the other material was the distractor; low/high refers to those trials in which the first item listed was the distractor and the second material was the target. Total refers to the percentage of correct responses for the combinations listed in the first column. 


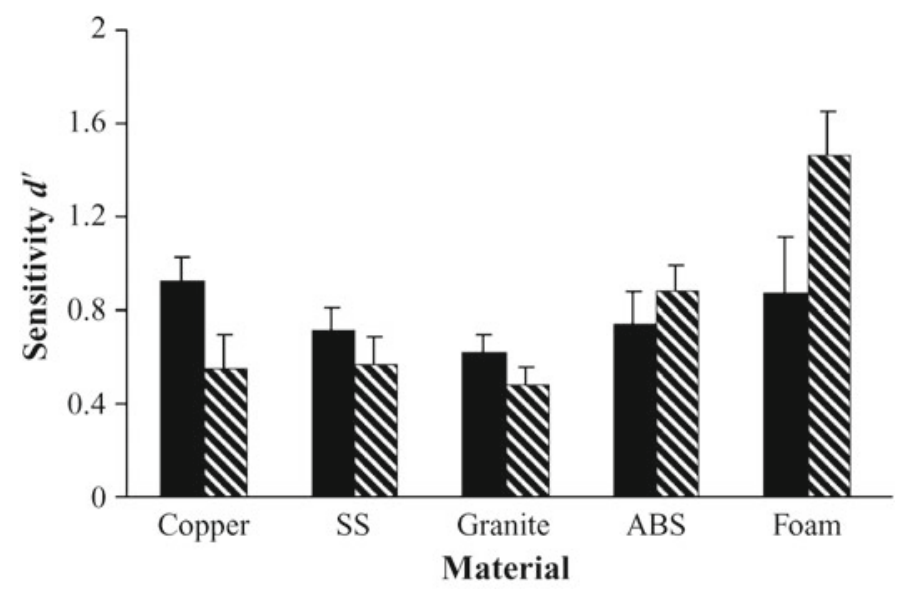

Figure 5. Group mean sensitivity $\left(d^{\prime}\right)$ in identifying the location of the target as a function of whether a material was a target or a distractor. Trials in which the material was a target are shown in black, and those in which the material was the distractor are striped. The error bars show the standard error of the mean. SS, stainless steel; ABS, a plastic.

sentation mode (target or distractor) as factors, indicated that there was a significant difference among the materials $[F(4,9)=11.92, p<.001]$ but no significant effect of presentation mode $[F(1,9)=1.90, p=.20]$. There was a significant interaction between materials and presentation mode $[F(4,36)=4.04, p=.008]$. The latter reflects the finding that distractors with low contact coefficients, such as foam and ABS, interfered less with identifying the target location than did distractors with high contact coefficients, such as copper and stainless steel.

It was of interest to determine whether performance was affected by the location of the target material - that is, whether it was presented to the index, middle, or ring finger. The group mean sensitivity, $d^{\prime}$, for each finger as a function of whether the target had a higher (high/low) or lower (low/high) contact coefficient than the distractor is shown in Figure 6. As can be seen in the figure, $d^{\prime}$ was lowest when the target was presented to the middle finger, especially when the target had a lower contact coefficient than did the distractor. A repeated measures ANOVA of these values, with presentation mode (high/low and low/ high) and fingers as factors, indicated that there was no significant effect of presentation mode $[F(1,9)=3.90$, $p=.08]$ and no significant difference among the three fingers $[F(2,9)=2.141, p=.15]$. There was also no significant interaction between fingers and presentation mode $(p=.50)$. These findings presumably reflect the high interindividual variability in this task.

\section{Discussion}

In this experiment, the subjects were required to identify which of three fingers was in contact with a material that was different from that in contact with the other two fingers. As in the first experiment, textural cues from the surface of the various materials were minimized so that the subjects would focus on the thermal responses on the fingertips when determining which material was the tar- get. A comparison of the performance of the subjects in the two experiments indicates that the second task was considerably more difficult than the first. For the same materials (i.e., copper, SS, ABS, and foam), the decline in performance when three, rather than two, fingers were involved was $26 \%$. This is not surprising, since in the second experiment, the subjects had to compare the thermal responses of three fingers, rather than two, and the stimuli were presented to the fingers of one hand, rather than to each hand separately. This result indicates that the effects of spatial summation across fingers within the hand have significantly reduced the ability of the subjects to discriminate between the thermal responses and, hence, to localize a target material.

The thermal properties of the materials were characterized by their contact coefficients, and performance was

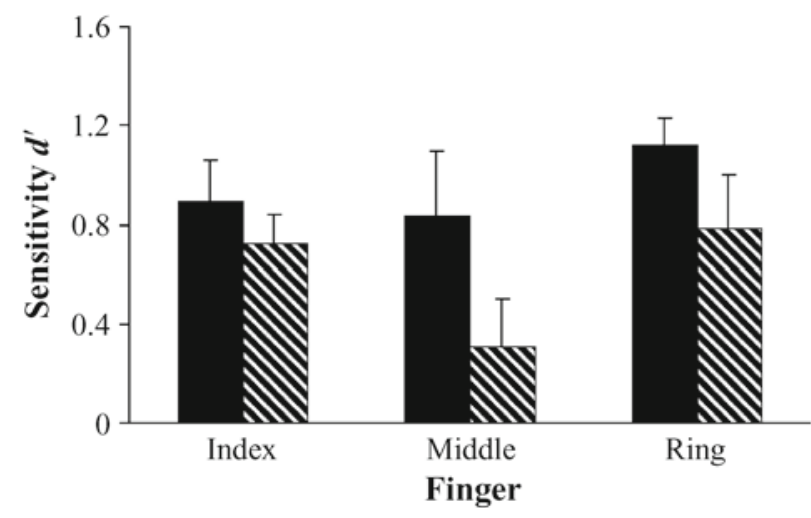

Figure 6. Group mean sensitivity $\left(d^{\prime}\right)$ in identifying the location of the target material for each finger. The means are calculated across the five materials presented and are shown as a function of whether the target presented to the finger was in a high/low (black) or a low/high (striped) combination. The error bars represent the standard error of the mean. 
then evaluated in terms of the ratio of the contact coefficients of the target and distractor materials. The ratio of the contact coefficients that is required to localize reliably a target in the high/low presentation mode is 14 , whereas it is 82 for the low/high mode. These findings show that it is easier to identify which finger is in contact with a target material when that finger is cooled more than the other fingers than when the two fingers are cooling more than the finger in contact with the target material. Although the change in skin temperature was not measured in this experiment, the results from the first experiment (see Figure 3) give some indication of the thermal responses of the fingers to contact with the various materials presented.

It is known that the perceived magnitude of cold sensation depends on the degree of cooling of the skin and on the areal extent of stimulation (Stevens \& Marks, 1979). Studies on the hand, forearm, back, and cheek have shown that there is pervasive spatial summation for cold, with the perceived magnitude of cold sensation depending almost as much on the size of the area of skin cooled as on the degree of skin cooling (Greenspan \& Kenshalo, 1985; Stevens \& Marks, 1979). In Stevens and Marks's experiment, the area of stimulation on the forearm was varied from 200 to $1,900 \mathrm{~mm}^{2}$, and the decrease in skin temperature ranged from $2^{\circ}$ to $12^{\circ} \mathrm{C}$, whereas Greenspan and Kenshalo applied thermal stimuli ranging from $0.1^{\circ}$ to $1^{\circ} \mathrm{C}$ to the thenar eminence of the hand and the area of stimulation varied from 50 to $700 \mathrm{~mm}^{2}$. In the present experiment, the decreases in skin temperature were similar to those in the latter study - probably between $0.1^{\circ}$ and $2^{\circ} \mathrm{C}$ - and the total area of contact on the finger pads was around $400 \mathrm{~mm}^{2}$. It is likely that spatial summation and, hence, poor localization of thermal changes on the fingers contributed to the inferior performance in this experiment, as compared with the material discrimination experiment.

The position of the target material was varied across trials in the present experiment, and it was predicted that target location would influence performance. This hypothesis was based on the findings of Green (1977) described earlier, in which temperature changes occurring at two adjacent fingers influenced the perceived magnitude of thermal sensation at the middle finger. In a further study of this phenomenon of thermal referral, Green (1978) found that referral of cold sensation was greatest between the middle and the ring fingers, as compared with the index and middle and the ring and little fingers, and that, for the latter fingers, it is generally quite small. In the context of the present experiment, the middle finger did perform more poorly than the other two fingers, although the differences between the fingers were not significant, due to the considerable variability between subjects.

\section{CONCLUSION}

In summary, the results from these two experiments indicate that subjects can discriminate between materials, using thermal cues, when the differences in the thermal properties of the materials are large. The ratio of the contact coefficients of the materials required for subjects to discriminate reliably between materials is substantially lower than the ratio necessary for subjects to identify a target material in the presence of two identical distractor materials. On the latter task, subjects found it easier to identify which finger was in contact with the target material when that finger was cooled more than the other two fingers than when the distractors resulted in a greater thermal change on the fingertips. These findings suggest, in agreement with earlier results (e.g., Green, 1977, 1978), that spatial summation across the fingers impairs the localization of cooling responses.

The decreases in skin temperature when contact was made with the materials were much smaller than those in the predictions calculated from the semi-infinite body model. This presumably reflects the thermal properties of both the skin - namely, that it is a good insulator-and of the vascular system in the finger, which is a source of heat. The changes in skin temperature appeared to be localized to the area of contact, and a more accurate measurement of the temperature changes in the finger would require a thermal imaging system. Future models of the thermal responses of the fingertips when different materials are contacted need to take into account the localized nature of the change and the effect of contact area and force on the thermal response.

\section{REFERENCES}

Caldwell, D. G., \& Gosney, C. (1993). Enhanced tactile feedback (tele-taction) using a multi-functional sensory system. Proceedings of the IEEE International Conference on Robotics \& Automation, 1, 955-960.

DARIAN-Smith, I. (1984). Thermal sensibility. In I. Darian-Smith (Ed.), Handbook of physiology: The nervous system. Sensory processes (Vol. III, pp. 879-913). Bethesda, MD: American Physiological Society.

Dyck, P. J., Curtis, D. J., BusheK, W., \& Offord, K. (1974). Description of "Minnesota thermal disks" and normal values of cutaneous thermal discrimination in man. Neurology, 24, 325-330.

EBERHART, R. C. (1985). Thermal models of single organs: Skin. In A. Shitzer \& R. C. Eberhart (Eds.), Heat transfer in medicine and biology (Vol. I, pp. 269-271). New York: Plenum.

Gescheider, G. A. (1997). Psychophysics: The fundamentals (3rd ed.). Mahwah, NJ: Erlbaum.

GreEN, B. G. (1977). Localization of thermal sensation: An illusion and synthetic heat. Perception \& Psychophysics, 22, 331-337.

GreEn, B. G. (1978). Referred thermal sensations: Warmth versus cold. Sensory Processes, 2, 220-230.

Greenspan, J. D., \& Kenshalo, D. R. (1985). The primate as a model for the human temperature-sensing system: 2 . Area of skin receiving thermal stimulation. Somatosensory Research, 2, 315-324.

HARDY, J. D., \& OPPEL, T. W. (1937). Studies in temperature sensation: III. The sensitivity of the body to heat and the spatial summation of the end organ responses. Journal of Clinical Investigation, 16, 533-540.

HARDY, J. D., \& OPPEL, T. W. (1938). Studies in temperature sensation: IV. The stimulation of cold sensation by radiation. Journal of Clinical Investigation, 17, 772-778.

Incropera, F. P., \& DeWitT, D. P. (1996). Fundamentals of heat and mass transfer. New York: Wiley.

Ino, S., Shimizu, S., Odagawa, T., Sato, M., Takahashi, M., Izumi, T., \& IfUKuBE, T. (1993). A tactile display for presenting quality of materials by changing the temperature of skin surface. In Proceedings of the 2nd IEEE International Workshop on Robot and Human Communication (pp. 220-224). New York: IEEE Press.

Johnson, K. O., Darian-Smith, I., \& LaMotte, C. (1973). Peripheral neural determinants of temperature discrimination in man: A correlative study of responses to cooling skin. Journal of Neurophysiology, 36, 347-370. 
Johnson, K. O., Darian-Smith, I., LaMotte, C., Johnson, B., \& OLDFIELD, S. (1979). Coding of incremental changes in skin temperature by a population of warm fibers in the monkey: Correlation with intensity discrimination in man. Journal of Neurophysiology, 42, 1332-1353.

Jones, L. A., \& BERrIs, M. (2003). Material discrimination and thermal perception. In Proceedings of the IEEE 11th International Symposium on Haptic Interfaces for Virtual Environment and Teleoperator Systems (pp. 171-178). New York: IEEE Press.

Kenshalo, D. R. (1976). Correlations of temperature sensitivity in man and monkey, a first approximation. In Y. Zotterman (Ed.), Sensory functions of the skin with special reference to man (pp. 305-330). Oxford: Pergamon.

LaMotte, R. H., \& Srinivasan, M. A. (1991). Surface microgeometry: Tactile perception and neural encoding. In O. Franzen \& J. Westman (Eds.), Information processing in the somatosensory system (pp. 4958). London: Macmillan.

Lederman, S. J., \& KlatzKy, R. L. (1997). Relative availability of surface and object properties during early haptic processing. Journal of Experimental Psychology: Human Perception \& Performance, 23, 1680-1707.

Lienhard, J. H., IV, \& LiEnhard, J. H., V (2003). A heat transfer textbook. Cambridge, MA: Phlogiston.

Marks, L. E., \& Stevens, J. C. (1973). Spatial summation of warmth: Influence of duration and configuration of the stimulus. American Journal of Psychology, 86, 251-267.

Marks, L. E., Stevens, J. C., \& Tepper, S. J. (1976). Interaction of spatial and temporal summation in the warmth sense. Sensory Processes, 1, 87-98.
Mills, A. F. (1999). Heat transfer (2nd ed.). Upper Saddle River, NJ: Prentice Hall.

Pawluk, D. T., \& Howe, R. D. (1999). Dynamic contact of the human fingerpad against a flat surface. Journal of Biomechanical Engineering, 121, 605-611.

Simmel, M. L., \& Shapiro, A. (1969). The localization of non-tactile thermal sensations. Psychophysiology, 5, 415-425.

Srinivasan, M. A., \& LaMotTe, R. H. (1995). Tactile discrimination of softness. Journal of Neurophysiology, 73, 88-101.

Stevens, J. C. (1991). Thermal sensibility. In M. A. Heller \& W. Schiff (Eds.), The psychology of touch (pp. 61-90). Hillsdale, NJ: Erlbaum.

Stevens, J. C., \& Choo, K. K. (1998). Temperature sensitivity of the body surface over the life span. Somatosensory \& Motor Research, 15, 13-28.

Stevens, J. C., \& Marks, L. E. (1979). Spatial summation of cold. Physiology \& Behavior, 22, 541-547.

Tubiana, R. T. (1981). Architecture and functions of the hand. In R. T. Tubiana (Ed.), The hand (pp. 19-93). Philadelphia: Saunders.

Verrillo, R. T., Bolanowski, S. J., Checkosky, C. M., \& McGlone, F. P. (1998). Effects of hydration on tactile sensation. Somatosensory \& Motor Research, 15, 93-108.

Wolfram-GABEL, R., \& SicK, H. (1995). Vascular networks of the periphery of the fingernail. Journal of Hand Surgery, 20B, 488-492.

(Manuscript received June 14, 2004; revision accepted for publication March 16, 2005.) 